\title{
The application of online Transcranial Random Noise Stimulation and Perceptual Learning in the improvement of visual functions in mild myopia
}

\author{
Rebecca Camilleri ${ }^{1}$, Andrea $\operatorname{Pavan}^{2}$ and Gianluca Campana ${ }^{1, *}$ \\ ${ }^{1}$ Dipartimento di Psicologia Generale, University of Padova, Italy; Human Inspired \\ Technology Research Centre - HIT, University of Padova, Italy. \\ ${ }^{2}$ School of Psychology, University of Lincoln, Brayford Pool, Lincoln LN2 1NB, UK.
}

Corresponding authors:

*Gianluca Campana

Dipartimento di Psicologia Generale

University of Padova

Via Venezia 8, 35131

Padova, Italy

Tel: +390498276651

Email: gianluca.campana@unipd.it 


\begin{abstract}
It has recently been demonstrated how perceptual learning, that is an improvement in a sensory/perceptual task upon practice, can be boosted by concurrent high-frequency transcranial random noise stimulation (tRNS). It has also been shown that perceptual learning can generalize and produce an improvement of visual functions in participants with mild refractive defects.

By using three different groups of participants (single-blind study), we tested the efficacy of a short training (8 sessions) using a single Gabor contrast-detection task with concurrent hftRNS in comparison with the same training with sham stimulation or hf-tRNS with no concurrent training, in improving visual acuity (VA) and contrast sensitivity (CS) of individuals with uncorrected mild myopia.

A short training with a contrast detection task is able to improve VA and CS only if coupled with hf-tRNS, whereas no effect on VA and marginal effects on CS are seen with the sole administration of hf-tRNS.

Our results support the idea that, by boosting the rate of perceptual learning via the modulation of neuronal plasticity, hf-tRNS can be successfully used to reduce the duration of the perceptual training and/or to increase its efficacy in producing perceptual learning and generalization to improved VA and CS in individuals with uncorrected mild myopia.
\end{abstract}

Keywords: mild myopia; visual perceptual learning; transcranial random noise stimulation; tRNS; contrast sensitivity; visual acuity 


\section{Introduction}

Sensory experience is important in shaping neural connections during the critical period early in life (Hubel \& Wiesel, 1970). Although this view still holds true, it has been challenged and reassessed by many authors, who have demonstrated that the visual system continues to mature and respond to environmental changes even in adulthood (Fregnac, Shulz, Thorpe, \& Bienenstock, 1988; Karni \& Sagi, 1991; Sale, et al., 2011). This notion of visual network plasticity is paramount not only in helping us achieve a better understanding of the human visual system and of visual plasticity mechanisms, but also in identifying noninvasive treatment tools and protocols to provide visual rehabilitation following injury, such as hemianopia following stroke (Huxlin, et al., 2009; Kasten, Wust, Behrens-Baumann, \& Sabel, 1998), amblyopia, and even when the visual deficit is at the non-cortical level such as in cases of myopia and presbyopia. In the latter case especially, being able to manipulate neuroplasticity might help us achieve visual recovery through compensatory strategies (Camilleri, Pavan, Ghin, Battaglini, \& Campana, 2014; Camilleri, Pavan, Ghin, \& Campana, 2014; Casco, et al., 2014; Durrie \& McMinn, 2007; Tan \& Fong, 2008).

To date, the most common non-invasive, behavioral method implemented to boost visual network plasticity and achieve recovery of function in a variety of visual disorders is known as Perceptual Learning (PL). Visual PL is the improvement on a visual task following repeated practice on the same or on a related task. (Levi \& Li, 2009; Nitsche, et al., 2009; Polat, 2009; Sagi, 2011). In the past decade, PL has started to be considered a useful tool for improving visual functions in clinical populations due to its generalizable effects. In fact, under specific conditions, PL is able to transfer to other stimuli, tasks and circumstances (Camilleri, Pavan, Ghin, Battaglini, et al., 2014; Jeter, Dosher, Petrov, \& Lu, 2009; Liu \& Weinshall, 2000; Maniglia, et al., 2011; McGovern, Webb, \& Peirce, 2012; Webb, Roach, \& McGraw, 2007; Xiao, et al., 2008; Zhou, et al., 2006), making it a practical tool that may yield potential benefits for various types of visual impairments. In general, most studies point to a localized increase in processing efficiency in $\mathrm{V} 1$ following practice on a visual perceptual task. This change can be attributed to a specific and localised plasticity in V1 or alternatively to a more complex network involving changes in the inputs $\mathrm{V} 1$ receives from other higher order brain regions following practice (top-down modulation). Additionally, PL may maximize in decision mechanisms the read-out of sensory signals through internal reward (Kumano \& Uka, 2013; Lu, Liu, \& Dosher, 2010; Petrov, Dosher, \& Lu, 2005).

So far, PL has been shown to be effective in improving, among other dysfunctions, visual abilities in amblyopia (Campana, Camilleri, Pavan, Veronese, \& Lo Giudice, 2014; 
Hussain, Webb, Astle, \& McGraw, 2012; Levi \& Li, 2009; Li, Young, Hoenig, \& Levi, 2005; Polat, 2009; Polat, Ma-Naim, Belkin, \& Sagi, 2004; Zhou, et al., 2006), mild refractive defects like myopia (Camilleri, Pavan, Ghin, Battaglini, et al., 2014; Tan \& Fong, 2008) and presbyopia (Polat, et al., 2012), and central or peripheral vision loss and cortical blindness (Chung, 2011; Das, Tadin, \& Huxlin, 2014; Huxlin, et al., 2009; Kasten, et al., 1998; Plank, et al., 2014; Rosengarth, et al., 2013; Sabel, Kenkel, \& Kasten, 2005). In myopia, visual input is limited by an optical de-focus, despite normal neuronal connectivity and image processing. In individuals with myopia, high spatial frequencies (SFs) are perceived as low contrast even when their physical contrast is high, resulting in a reduced visual acuity (VA) (Durrie \& McMinn, 2007; Tan \& Fong, 2008). Naturally, in the case of refractive defects, sensory training does not modify the structure of the eye; nonetheless improvements on uncorrected (that is: in absence of any optical correction) VA and contrast sensitivity (CS) using perceptual learning paradigms have been found; the proposed underlying mechanism in the case of non-cortical visual defects is an increase of signal-to-noise ratio of the de-focused retinal image (Durrie \& McMinn, 2007; Tan \& Fong, 2008). PL with the use of single Gabor stimuli, has been shown to be able to increase VA and CS in a clinical population of patients with amblyopia (Zhou, et al., 2006), and in recent studies conducted by Camilleri, Pavan, Ghin, Battaglini, et al. (2014) and Casco et al. (2014) it was found that PL using a simple contrast detection task is able to improve uncorrected VA in participants with mild myopia.

Despite its proven effectiveness, PL techniques require lengthy protocols in order to yield effective outcomes (usually a minimum of two months training of up to three to four times weekly) (Camilleri, Pavan, Ghin, Battaglini, et al., 2014; Polat, et al., 2004; Tan \& Fong, 2008). Random noise stimulation could optimize the effects of a behavioral training with measurable changes in the brain by modulating neuronal excitability that are involved in long-term potentiation (Fritsch, et al., 2010; Stagg, et al., 2009) which may ultimately lead to neuroplasticity. Recent advancements in the field have highlighted the potential benefits of non-invasive brain stimulation (NIBS) techniques. These techniques allow for external modulation of neural activity in the human brain. Besides transcranial magnetic stimulation (TMS), the most common NIBS technique for improving perceptual or cognitive functions is transcranial direct current stimulation (tDCS), whereby a low constant current is delivered to the brain areas of interest via electrodes (an anode and a cathode) positioned on the scalp. Cortical excitability is typically increased under the anode, and decreased under the cathode (Nitsche \& Paulus, 2000). tDCS has been shown to successfully improve various perceptual or cognitive abilities both in healthy participants and in patients, and when administered 
either with (Looi, Duta, Brem, Huber, Nuerk \& Cohen Kadosh, 2016) or without a concurrent training (Coffman, Clark \& Parasuraman, 2014). In particular, tDCS has been found to

improve functions such as working memory (Andrews, Hoy, Enticott, Daskalakis \&

Fitzgerald, 2011; Fregni, Boggio, Nitsche, Bermpohl, Antal, Feredoes, Marcolin, Rigonatti,

Silva, Paulus \& Pascual-Leone, 2005), long-term memory (Marshall, Molle, Hallschmid \&

Born, 2004; Sandrini, Brambilla, Manenti, Rosini, Cohen \& Cotelli, 2014), language

(Fertonani, Rosini, Cotelli, Rossini \& Miniussi, 2010; Fiori, Coccia, Marinelli, Vecchi,

Bonifazi, Ceravolo, Provinciali, Tomaiuolo \& Marangolo, 2010; Monti, Cogiamanian,

Marceglia, Ferrucci, Mrakic-Sposta, Vergari, Zago \& Priori, 2008; Pisoni, Papagno \&

Cattaneo, 2012; Ross, McCoy, Wolk, Coslett \& Olson, 2010; Sparing, Dafotakis, Meister,

Thirugnanasambandam \& Fink, 2008), visual and attentional skills (Antal, Nitsche, Kruse,

Kincses, Hoffmann \& Paulus, 2004; Bolognini, Fregni, Casati, Olgiati \& Vallar, 2010; Kraft,

Roehmel, Olma, Schmidt, Irlbacher \& Brandt, 2010; Ding, Li, Spiegel, Chen, Chan, Luo,

Yuan, Deng, Yu \& Thompson, 2010). However, efficacy of tDCS has recently been disputed

(e.g.: Mancuso, Ilieva, Hamilton \& Farah, 2016; Horvath, Forte \& Carter, 2015; Vannorsdall, van Steenburgh, Schretl, Jayatillake, Skolasky \& Gordon, 2016).

Transcranial random noise stimulation (tRNS) is an innovative method for boosting neural plasticity through the application of a weak alternating current at random frequencies $(0.1-640 \mathrm{~Hz})$. The neuromodulatory effects of tRNS are said to facilitate or inhibit neuronal activity by syncronising or desyncronising it (Grenier, Timofeev, \& Steriade, 2001; Moss, Ward, \& Sannita, 2004; Ponomarenko, Li, Korotkova, Huston, \& Haas, 2008). Unlike in transcranial direct current stimulation (tDCS), where the neurons are embedded in a constant electrical field and may result in a homeostatic effect of the ion neural channels after prolonged use, random noise stimulation counteracts this phenomenon due to its fluctuating pattern. In fact, one known disadvantage of continuous use of tDCS for rehabilitative purposes is that it may result in a homeostatic effect of the neural population being stimulated, in that neurons tend to return to their initial 'resting state'. This counteracting effect of tRNS may be attributed either to the repeated opening of sodium channels or to a higher sensitivity of neuronal networks to field modulation than the average single neuron threshold (Terney, Chaieb, Moliadze, Antal, \& Paulus, 2008).

Unlike tDCS, tRNS has only recently been explored within the visual domain (Camilleri, Pavan, Ghin, Battaglini, et al., 2014; Campana, et al., 2014; Fertonani, Pirulli, \& Miniussi, 2011; Pirulli, Fertonani, \& Miniussi, 2013). Fertonani and colleagues conducted a study testing the efficacy of different stimulation protocols on an orientation discrimination 
task (Fertonani, et al., 2011). Their results concluded that high frequency tRNS (hf-tRNS), that is tRNS with a frequency ranging from 100 to $640 \mathrm{~Hz}$, was the most efficacious type of NIBS for boosting PL in healthy participants, when compared with low frequency random noise, direct current (anodal and cathodal) and sham stimulation. Other studies show tRNS as yielding faster and more effective PL as well as transfer to VA and CS in healthy participants, people with mild myopia and amblyopia (Camilleri, Pavan, Ghin, \& Campana, 2014; Campana, et al., 2014). In particular, a preliminary study showed that applying tRNS with a PL training protocol of 2 weeks ( 8 sessions) is able to achieve the same functional outcome on uncorrected VA, and a better outcome on uncorrected CS, as a two-month training protocol (24 sessions) (Camilleri, Pavan, Ghin, Battaglini, et al., 2014; Camilleri, Pavan, Ghin, \& Campana, 2014).

The aforementioned studies were unable to determine whether it is specifically the combined use of the techniques that brought about this fast improvement or whether tRNS alone is able to achieve the same outcome on the visual system in the absence of any behavioural training or whether there may be any potential placebo effect of the electrical stimulation. By using a between-groups approach where participants are trained, using a contrast detection training (Camilleri, Pavan, Ghin, Battaglini, et al., 2014; Camilleri, Pavan, Ghin, \& Campana, 2014; Zhou, et al., 2006), with concurrent tRNS, or sham stimulation, or else receive tRNS with no behavioural training, the aim of this study is to isolate the contribution of PL, tRNS and the combination of both. Furthermore, this work focuses on the potential application of Transcranial Electrical Stimulation (TES), specifically tRNS, together with behavioral trainings, as a new approach to further ameliorate visual outcomes of existing training paradigms.

\section{Methods}

\section{Apparatus}

Both the behavioural training and pre/post-tests were displayed on a 22-inch Philips Brilliance 202P4 luminance-calibrated (gamma-corrected with gamma =1) monitor with a refresh rate of $60 \mathrm{~Hz}$. The stimuli used in the training were created with the Matlab Psychtoolbox (Brainard, 1997; Pelli, 1997), whereas stimuli for measuring VA in the pre and post-tests were generated using the Freiburg Acuity and Contrast Test (FrACT 3.8) (Bach, 1996, 2007). Spatial dithering (Bach, 1997) and colour bit stealing (Tyler, 1997) for increasing the depth of contrast resolution (12 bits) were enabled on the FrACT, thus allowing 
higher luminance resolution. The screen resolution was 1280x1024 pixels, each pixel subtended 0.33 arcmin at a viewing distance of 3 meters, and 0.67 arcmin at a viewing distance of 1.5 meters. Viewing distance was equal to 3 meters for pre- and post-tests, whereas the training was administered from 1.5 meters (Das, et al., 2014; McGovern, et al., 2012). All stimuli were presented centrally and both the tests and training were carried out in a dark, silent room. Background screen luminance (corresponding to mean luminance of Gabor stimuli) was $31.5 \mathrm{~cd} / \mathrm{m}^{2}$.

\section{Transcranial Random Noise Stimulation (tRNS)}

The high frequency transcranial random noise stimulation was delivered to groups 1 and 3 using a battery-driven stimulator (BrainSTIM, EMS) through a pair of saline-soaked sponge electrodes. The tRNS consisted of an alternating current of $1.5 \mathrm{~mA}$ intensity with a 0mA offset applied at random frequencies. The frequencies ranged from 100 to $640 \mathrm{~Hz}$ (high frequency range). This stimulation protocol has been demonstrated efficacious in boosting perceptual learning in previous studies (Camilleri, Pavan, Ghin, Battaglini, et al., 2014; Fertonani, et al., 2011; Pirulli, et al., 2013). The active electrode had an area of $16 \mathrm{~cm}^{2}$ and was placed over the occipital cortex measured at $\sim 3 \mathrm{~cm}$ above the inion. The reference electrode had an area of $60 \mathrm{~cm}^{2}$ and was placed on the forehead. The current density was always maintained below the safety limits (below $1 \mathrm{~A} / \mathrm{m}^{2}$ ) (Poreisz, Boros, Antal, \& Paulus, 2007). The electrodes were kept in place with bandages.

\section{Participants}

Thirty participants with mild myopia were recruited from the University of Padova (mean age of 25.31, ranging between 19 and 29). The participants were randomly assigned to one of three different treatment groups each consisting of 10 participants. The first group (PL+tRNS) carried out a 2-week (8 sessions) behavioral training using a contrast detection task combined with online high frequency tRNS (hf-tRNS) for 25 minutes of stimulation. The second group (PL+Sham) took part in the same training protocol but combined with Sham stimulation. The third group (tRNS alone) of participants carried out 25 minutes of hf-tRNS in the absence of any behavioral training. This was done in order to compare the effects of combining behavioral training with tRNS with the effects of behavioral training alone (without tRNS) and tRNS alone on uncorrected VA and uncorrected CS.

All participants fit the following inclusion criteria: refractive error up to -2 diopters (D) in either eye (minimum was $-0.75 \mathrm{D}$ ), with astigmatism not exceeding $-0.5 \mathrm{D}$ in either eye. 
All tests and the behavioral training were administered binocularly and without the use of optical corrections. All participants had a stable refractive index for the 6 months preceding the training. Exclusion criteria included the presence of any other condition for reduced VA other than simple myopia and/or mild astigmatism, including pregnancy, diabetes mellitus, presence of myopia-related ocular complications and any previous ocular surgery. To ensure the inclusion and exclusion criteria were met, prior to commencement of the training, the participants carried out a detailed assessment by an optometrist. Additionally, each participant in the two tRNS groups filled in a questionnaire to check that all were eligible to undergo non-invasive brain stimulation (e.g., no history of seizures, no internal metal objects or previous traumatic brain injury). At the end of the study each participant was given the possibility to participate to the protocol that produced the largest mean improvement in VA. The Local Ethics Committee approved this study.

\section{Stimuli and Procedure}

Prior to (pre-tests) and after the training (with or without tRNS) (post-tests), uncorrected VA and uncorrected CS were measured for each participant by using Landolt-C and Grating tests of the Freiburg Visual Acuity Test (FrACT) (Bach, 1996, 2007). The BestPest adaptive procedure was used to calculate the absolute threshold for each of these tests. Stimulus duration lasted until the participants' response. An auditory cue was presented upon stimulus presentation and a different auditory cue was implemented as feedback for error responses.

The Landolt-C test was used to assess uncorrected VA. The task of the participants was to indicate, in every trial, the orientation of the gap of the Landolt-C out of eight possible orientations. Contrast stimuli for the uncorrected CS assessment consisted of sinusoidal gratings presented in a circular window with a narrow Gaussian taper. Size of the gratings was 3 deg. Grating orientations used were $0,45,90$ or $135 \mathrm{deg}$. The task of the participant was to discriminate the orientation of the grating at different spatial frequencies, ranging from 1 to 15 cycles per degree (cpd), in separate blocks.

The behavioral (training) paradigm consisted of a two-interval forced choice (2IFC) task where the participants had to detect the presence of a single Gabor patch, which changed in contrast according to the performance of the participant. The threshold corresponding to $79 \%$ of correct detection was determined by using a 1up-3down staircase procedure(Levitt, 1971). Stimuli used in the training were comprised of Gabor patches consisting of a cosinusoidal carrier enveloped by a stationary Gaussian. Standard deviation of the luminance 
Gaussian envelope $(\sigma)$ was equal to the sinusoidal wavelength $(\lambda)$; therefore, the size of the Gabor patch covaried with its spatial frequency. Additionally, the spatial phase of the cosinusoidal carrier equaled to zero (evenly symmetric Gabor patch). Stimulus duration lasted $200 \mathrm{~ms}$. In order to reduce spatial and temporal uncertainty both an auditory and a spatial cue were implemented. On each trial a central fixation point preceded the presentation of each interval, and an auditory cue indicated when the stimulus (if present) appeared. Performance feedback was also provided to the participants in the form of an auditory beep following an incorrect response.

Participants in groups 1 and 2 (PL+tRNS and PL+Sham respectively) underwent 8 training sessions over 2 weeks ( 4 consecutive sessions each week) and trained on 4 different orientations of the stimulus with a single spatial frequency (ranging from 6 to $15 \mathrm{cpd}$ ) chosen according to the individual's cutoff performance in the pretest uncorrected CS measurement, defined as the spatial frequency at which the estimated contrast threshold from pre-training uncorrected CS measurements was closest to 0.50 (Michelson contrast) (Zhou, et al., 2006). Since interleaving different stimulus conditions (roving) has been shown to hinder perceptual learning (Herzog, Aberg, Fremaux, Gerstner, \& Sprekeler, 2012; Kuai, Zhang, Klein, Levi, \& $\mathrm{Yu}, 2005)$, in order to increase the efficacy of perceptual learning, participants were trained on the same orientation for 2 consecutive days. Each session consisted of 8 blocks each containing 60 trials, which lasted for approximately 45 minutes.

Participants in group 1 (PL+tRNS) were administered hf-tRNS (1.5mA) during the first 25 minutes of each session, which covered the first 5 blocks (Fertonani, et al., 2011). Participants in group 2 (PL+Sham) underwent sham stimulation for the same length of time. In the sham condition, the stimulation was a placebo for all eight blocks and was delivered for 20s at the beginning of each block. Participants in group 3 (tRNS alone) were administered hf-tRNS (1.5mA) for 25 minutes, without any concurrent task, for 8 sessions over 2 weeks (4 consecutive sessions each week). This matches the stimulation parameters of group 1.

\section{Results}

All data were subject to the Kolmogorov-Smirnov test of normality. Pre- and posttests of uncorrected VA measurements, for each of the three groups, were normally distributed ( $p>.05)$, therefore ANOVAs and t-tests were used for this data. For what concerns Log-transformed uncorrected CS, pre- and post-tests with various spatial frequencies in the three groups, were not normally distributed $(\mathrm{p}<.05)$, therefore the non-parametric Wilcoxon 
signed-rank test was used, separately for each group and for each spatial frequency, to assess differences between pre- and post-tests.

Uncorrected VA at pre-test underwent to a one-way ANOVA in order to assess whether there were any significant VA differences between groups that could in turn explain different training/stimulation outcomes. No significant differences were found between the three groups in uncorrected VA at pre-test $\left(\mathrm{F}_{2,27}=0.09, \mathrm{p}>.05, \eta_{\mathrm{p}}^{2}=0.007\right)$.

A mixed-design ANOVA with 'group' as the between subjects factor was used to compare pre- and post-test measurements of uncorrected VA. A significant interaction of pre/post by group indicated that the groups differed in their pre versus post outcome $\left(\mathrm{F}_{2,27}=14.481, \mathrm{p}<.0001, \eta_{\mathrm{p}}^{2}=0.518\right)$. Post hoc analysis was carried out using $\mathrm{t}$-tests which revealed a significant difference for pre and post-test in group $1(\mathrm{PL}+\mathrm{tRNS})\left(\mathrm{t}_{9}=4.474, \mathrm{p}<.01\right.$, $\mathrm{r}=0.83$ ). Results indicate an improvement of $0.171 \mathrm{LogMAR}$ from a baseline measurement of 0.337 LogMAR to a post-test reading of 0.166 LogMAR (Figure 1). No significant differences were observed for either group 2 (PL+Sham, $\mathrm{t}_{9}=-1.221, \mathrm{p}>.05, \mathrm{r}=0.37$ ) or group 3 (tRNS alone, $\mathrm{t}_{9}=-0.295, \mathrm{p}>.05, \mathrm{r}=0.09$ ). Bonferroni-corrected $\mathrm{t}$-tests aiming at comparing the amount of improvement in the different groups revealed a significant difference between group 1 and $2\left(\mathrm{t}_{18}=4.36, \mathrm{p}<.001, \mathrm{r}=0.71\right)$ and between group 1 and $3\left(\mathrm{t}_{18}=4.15, \mathrm{p}<.01, \mathrm{r}=0.7\right)$, but not between group 2 and $3\left(\mathrm{t}_{18}=-0.83, \mathrm{p}>.05, \mathrm{r}=0.19\right)$.

\section{FIGURE 1 ABOUT HERE}

With regards to uncorrected CS, pre- and post-test measurements were compared with a Wilcoxon signed-rank test on the Log-transformed CS data. Participants undergoing both random noise stimulation and behavioural training (group 1) improved significantly at the following spatial frequencies: 3 cpd $(Z=-1.988, p<.05), 5$ cpd $(Z=-2.293, p<.05), 7$ cpd $(Z=-$ $2.09, \mathrm{p}<.05), 9 \mathrm{cpd}(\mathrm{Z}=-2.191, \mathrm{p}<.05)$, and $11 \mathrm{cpd}(\mathrm{Z}=-2.599, \mathrm{p}<.01)$. No significant improvements were seen at the lowest $(1 \mathrm{cpd})$ and highest $(15 \mathrm{cpd})$ tested spatial frequencies (Figure 2). In group 2 (PL+Sham), no significant differences were observed at any tested special frequency. Interestingly in group 3, which included only tRNS, a significant difference was found at $9 \mathrm{cpd}(\mathrm{Z}=-1.988, \mathrm{p}=<.05)$. No other significant differences were found in group 3. 


\section{Discussion}

The present study focuses on the application of tRNS together with a visual training, specifically, a single Gabor contrast detection paradigm (Camilleri, Pavan, Ghin, \& Campana, 2014; Zhou, et al., 2006), to further enhance the outcome of existing PL regimes so as to improve visual defects. In this study, however, we did not find any improvement in either VA or CS when the visual training was administered in absence of tRNS (PL+Sham group). This could be possibly due to the failure of achieving PL with only 8 sessions where orientation was changed every two sessions, similar to a roving procedure (Herzog, et al., 2012). In fact, by using the same PL paradigm (contrast detection) in myopic participants, in a previous study we found that 24 sessions were needed to achieve a VA improvement of 0.16 LogMAR, whereas no improvement in CS was found even with such a long training (Camilleri, Pavan, Ghin, Battaglini, et al., 2014). However, if PL is boosted by the concomitant administration of hf-tRNS as shown by previous studies (Camilleri, Pavan, Ghin, Battaglini, et al., 2014; Campana, et al., 2014; Fertonani, et al., 2011; Pirulli, et al., 2013), then 8 sessions of training become enough efficacious to improve uncorrected VA of a similar amount of 24 training sessions with no concurrent stimulation, and to significantly improve uncorrected CS (whereas no improvement was obtained with 24 training sessions using the same paradigm (Camilleri, Pavan, Ghin, Battaglini, et al., 2014).

The obtained improvements in uncorrected VA and uncorrected CS in the combined treatment group suggest a specific mechanism underlying the effects by which tRNS acts: it seems to require external sensory (visual) input, thereby acting on the neurons activated by the task at hand. This idea is further strengthened by the lack of significant improvement when tRNS was applied in isolation of a behavioural task. Interestingly however, in the absence of the behavioural training, tRNS resulted in improved contrast sensitivity at $9 \mathrm{cpd}$. Some improvement in contrast sensitivity with the sole use of brain stimulation, so far, has been observed in individuals with amblyopia after the administration of either anodal tDCS (Spiegel, Byblow, Hess, \& Thompson, 2013) or high-frequency repetitive TMS (Clavagnier, Thompson, \& Hess, 2013; Thompson, Mansouri, Koski, \& Hess, 2008). Although here, improvement on CS is much more limited (to a single spatial frequency) than when combined with PL, suggesting a weaker effect of tRNS in the absence of a behavioural task. These results may be explained by the underlying phenomenon of stochastic resonance, whereby the signal to noise ratio of the low threshold stimulus may be enhanced by adding noise to the system (Terney, et al., 2008). Stochastic resonance has been used previously for explaining enhancements of behavioural effects that have been found with TMS, therefore moving 
beyond the older idea of TMS as a "virtual lesion": if a low level of neural noise (produced by low-intensity TMS) is introduced into the system during processing of a weak stimulus, the membrane potential of those neurons tuned to the stimulus will be increased so to reach the threshold and make them firing, consequently, stimulus detection/discrimination will be facilitated (Miniussi, Harris \& Ruzzoli, 2013; Schwarzkopf, Silvanto \& Rees, 2011). In the present study, the random noise stimulation at frequencies between 100 and $640 \mathrm{~Hz}$ may be interacting with the neurons already activated by the low contrast Gabor stimuli and by doing so, enhance their firing response (increasing signal to noise ratio), whilst preventing the network from becoming desensitized and progress to a homeostatic state as can occur with tDCS. On the other hand, when tRNS is applied in the absence of any input, the stimulation is unspecific and may simply add noise to the system. In fact, there is emerging consensus that the effects of brain stimulation techniques are highly dependent on the state of the stimulated neuronal population (Cattaneo \& Silvanto, 2008; Silvanto, Muggleton, Cowey \& Walsh, 2007; Silvanto, Muggleton, \& Walsh, 2008). For example, in a study investigating the effects of offline theta burst stimulation on a subsequent visual motion discrimination task, Silvanto and colleagues (Silvanto, Muggleton, Cowy \& Walsh, 2007) found that external induction of neuronal plasticity (such as in the case of brain stimulation) also depends on the state of the targeted neurons during stimulation. Despite here induction of neural plasticity was found with above-threshold (simple) stimuli, larger improvements in tasks via perceptual learning (and thus neural plasticity) typically occur with more challenging, near-threshold stimuli (Polat et al., 2004; Sagi, 2011).

Since the seminal paper of Bliss and Lomo (1973), it is well established that high frequency stimulation is able to produce long term potentiation through strengthening of synaptic connections. More recently, it has been suggested that also noisy electrical fluctuations are able to boost synaptic signals (Moss, et al., 2004). Interestingly, oscillations within a frequency range of $80-200 \mathrm{~Hz}$ included in the high frequency band, have been associated with plasticity processes (Grenier, et al., 2001) and learning (Ponomarenko, et al., 2008). Another recent study by Fertonani and colleagues (Fertonani, et al., 2011) explains how the repetitive action of tRNS may induce direct temporal summation of neural activity and may desynchronize (pathological or inefficient) rhythms by increasing the signal to noise ratio. A very recent study proposes that, unlike tDCS, tRNS-induced plasticity is independent of NMDA receptors and involves the modulation of voltage-gated sodium channels (Chaieb, Antal, \& Paulus, 2015). Due to the recurring potentiation of sodium channels, its aftereffects through LTP may outlast those observed after tDCS stimulation. The aftereffects of tRNS on 
cortical excitability have recently been evaluated in the motor cortex by measuring the participants MEPs following 4, 5 and 6 minutes of stimulation (Chaieb, Paulus, \& Antal, 2011). The researchers observed that increased cortical excitability following 5 minutes of tRNS lasted only for 10 minutes. Whereas 6 minutes of tRNS induced an even stronger excitability increase of up to 30 minutes post stimulation.

The application of TES as a potential tool in neuro-rehabilitation is a relatively young concept. Yet many studies are seeking to understand the mechanisms by which different TES techniques can complement an existing cognitive training (Dhaliwal, Meek, \& Modirrousta, 2015; Yun, Chun, \& Kim, 2015). tES is non-invasive and if used correctly should not lead to any aversive effects, it is relatively cheap and can be implemented in various contexts as an adjunct to existing techniques, which although are effective in isolation, might not be offering the most optimal treatment to patients. tRNS, a younger sister of tDCS, has not featured in as many studies, yet due to the mechanisms by which it acts and its lack of discomfort, is starting to capture the attention of many clinical researchers. The present study identifies the potential this device has in assisting existing visual rehabilitation methods, such as PL, and encourages further insight into the exact mechanisms by which it is acting. In line with the present work, a recent study by Campana and colleagues, suggested how hf-tRNS stimulation applied to V1 in combination with a lateral masking paradigm results in a significant improvement in VA and CS in the amblyopic eye of participants (Campana, et al., 2014). Furthermore, the improvements following combined tRNS and PL in mild myopia, using the same protocol as in the present study, have been shown to be maintained for up to 3 months post training (Camilleri, Pavan, Ghin, Battaglini, et al., 2014).

Following these positive results, a larger clinical study is paramount in order to investigate more reliably, the effectiveness of these techniques in other clinical populations. In addition, it is necessary that follow-up measures are taken post-training to establish long term effects while allowing for flexible re-application of the training. It is still unclear what relevance these improvements will have in a real-life setting outside the laboratory. Additional use of questionnaires and self-reports assessing day-to-day improved vision is essential. 


\section{Figure captions}

Figure 1. Mean uncorrected VA improvement (LogMAR difference) between pre- and posttest is shown for each of the three groups of participants. Error bars \pm 1 SEM.

Figure 2. Mean uncorrected CS improvement (Log-transformed difference) between pre- and post-test is shown for each tested spatial frequency, separately for each of the three groups of participants. Error bars \pm 1 SEM.

\section{Acknowledgements}

GC was supported by Progetto di Ateneo 2014 (CPDA148575/14) funded by the University of Padova. RC was supported by a CARIPARO PhD fellowship. 


\section{References}

Andrews, S. C., Hoy, K. E., Enticott, P. G., Daskalakis, Z. J., \& Fitzgerald F. B. (2011). Improving working memory: the effect of combining cognitive activity and anodal transcranial direct current stimulation to the left dorsolateral prefrontal cortex. Brain Stimul, 4(2), 84-89.

Antal, A., Nitsche, M.A., Kruse, W., Kincses, T.Z., Hoffmann, K.P., \& Paulus, W. (2004). Direct current stimulation over V 5 enhances visuo-motor coordination by improving motion perception in humans. J Cogn Neurosci, 16, 521-527.

Bach, M. (1996). The Freiburg Visual Acuity Test-automatic measurement of visual acuity. Optometry Vision Science, 73, 49-53.

Bach, M. (1997). Anti-aliasing and dithering in the 'Freiburg Visual Acuity Test'. Spat Vis, 11, 85-89.

Bach, M. (2007). The Freiburg Visual Acuity Test-variability unchanged by post-hoc reanalysis. Graefes Arch Clin Exp Ophthalmol, 245, 965-971.

Bliss, T. V., \& Lomo, T. (1973). Long-lasting potentiation of synaptic transmission in the dentate area of the anaesthetized rabbit following stimulation of the perforant path. J Physiol, 232, 331-356.

Bolognini, N., Fregni, F., Casati, C., Olgiati, E., \& Vallar, G. (2010). Brain polarization of parietal cortex augments training-induced improvement of visual exploratory and attentional skills. Brain Res, 1349, 76-89.

Brainard, D. H. (1997). The Psychophysics Toolbox. Spat Vis, 10, 433-436.

Camilleri, R., Pavan, A., Ghin, F., Battaglini, L., \& Campana, G. (2014). Improvement of uncorrected visual acuity and contrast sensitivity with perceptual learning and transcranial random noise stimulation in individuals with mild myopia. Front Psychol, 5, 1234.

Camilleri, R., Pavan, A., Ghin, F., \& Campana, G. (2014). Improving myopia via perceptual learning: is training with lateral masking the only (or the most) efficacious technique? Atten Percept Psychophys, 76, 2485-2494.

Campana, G., Camilleri, R., Pavan, A., Veronese, A., \& Lo Giudice, G. (2014). Improving visual functions in adult amblyopia with combined perceptual training and transcranial random noise stimulation (tRNS): a pilot study. Front Psychol, 5, 1402.

Casco, C., Guzzon, D., Moise, M., Vecchies, A., Testa, T., \& Pavan, A. (2014). Specificity and generalization of perceptual learning in low myopia. Restor Neurol Neurosci, 32, 639-653.

Cattaneo, Z, \& Silvanto, J. (2008). Time course of the state-dependent effect of transcranial magnetic stimulation motion in the TMS-adaptation paradigm. Neuroscience Letters, 443, 82-85.

Chaieb, L., Antal, A., \& Paulus, W. (2015). Transcranial random noise stimulationinduced plasticity is NMDA-receptor independent but sodium-channel blocker and benzodiazepines sensitive. Front Neurosci, 9, 125.

Chaieb, L., Paulus, W., \& Antal, A. (2011). Evaluating aftereffects of short-duration transcranial random noise stimulation on cortical excitability. Neural Plast, 2011, 105927.

Chung, S. T. (2011). Improving reading speed for people with central vision loss through perceptual learning. Invest Ophthalmol Vis Sci, 52, 1164-1170.

Clavagnier, S., Thompson, B., \& Hess, R. F. (2013). Long lasting effects of daily theta burst rTMS sessions in the human amblyopic cortex. Brain Stimul, 6, 860-867. 
Coffman, B. A., Clark, V. P., \& Parasuraman, R. (2014). Battery powered thought: enhancement of attention, learning, and memory in healthy adults using transcranial direct current stimulation. Neuroimage, 85(3), 895-908.cof

Das, A., Tadin, D., \& Huxlin, K. R. (2014). Beyond blindsight: properties of visual relearning in cortically blind fields. J Neurosci, 34, 11652-11664.

Dhaliwal, S. K., Meek, B. P., \& Modirrousta, M. M. (2015). Non-Invasive Brain Stimulation for the Treatment of Symptoms Following Traumatic Brain Injury. Front Psychiatry, 6, 119.

Ding, Z., Li, J., Spiegel, D. P., Chen, Z., Chan, L., Luo, G., Yuan, J., Deng, D., Yu, M., \& Thompson, B. (2016). The effect of transcranial direct current stimulation on contrast sensitivity and visual evoked potential amplitude in adults with amblyopia. Sci Rep, 6:19280.

Durrie, D., \& McMinn, P. S. (2007). Computer-based primary visual cortex training for treatment of low myopia and early presbyopia. Trans Am Ophthalmol Soc, 105, 132-138; discussion 138-140.

Fertonani, A., Pirulli, C., \& Miniussi, C. (2011). Random noise stimulation improves neuroplasticity in perceptual learning. J Neurosci, 31, 15416-15423.

Fertonani, A., Rosini, S., Cotelli, M., Rossini, P. M., \& Miniussi, C. (2010). Naming facilitation induced by transcranial direct current stimulation. Behav Brain Res, 208(2), 311-318.

Fiori, V., Coccia, M., Marinelli, C. V., Vecchi, V., Bonifazi, S., Ceravolo, M. G., Provinciali, L., Tomaiuolo, F., \& Marangolo, P. (2010). Transcranial direct current stimulation improves word retrieval in healthy and nonfluent aphasic subjects. J Cogn Neurosci, 23(9), 2309-2323.

Fregnac, Y., Shulz, D., Thorpe, S., \& Bienenstock, E. (1988). A cellular analogue of visual cortical plasticity. Nature, 333, 367-370.

Fregni, F., Boggio, P. S., Nitsche, M. A., Bermpohl, F., Antal, A., Feredoes, E., Marcolin, M. A., Rigonatti, S. P., Silva, M. T., Paulus, W., \& Pascual-Leone, A. (2005). Anodal transcranial direct current stimulation of prefrontal cortex enhances working memory. Exp Brain Res, 166(1), 23-30.

Fritsch, B., Reis, J., Martinowich, K., Schambra, H. M., Ji, Y., Cohen, L. G., \& Lu, B. (2010). Direct current stimulation promotes BDNF-dependent synaptic plasticity: potential implications for motor learning. Neuron, 66, 198-204.

Grenier, F., Timofeev, I., \& Steriade, M. (2001). Focal synchronization of ripples (80-200 $\mathrm{Hz}$ ) in neocortex and their neuronal correlates. J Neurophysiol, 86, 1884-1898.

Herzog, M. H., Aberg, K. C., Fremaux, N., Gerstner, W., \& Sprekeler, H. (2012). Perceptual learning, roving and the unsupervised bias. Vision Res, 61, 95-99.

Horvath, J. C, Forte, J. D., \& Carter O. (2015). Quantitative Review Finds No Evidence of Cognitive Effects in Healthy Populations From Single-session Transcranial Direct Current Stimulation (tDCS). Brain Stimul, 8(3), 535-550.

Hubel, D. H., \& Wiesel, T. N. (1970). The period of susceptibility to the physiological effects of unilateral eye closure in kittens. J Physiol, 206, 419-436.

Hussain, Z., Webb, B. S., Astle, A. T., \& McGraw, P. V. (2012). Perceptual learning reduces crowding in amblyopia and in the normal periphery. J Neurosci, 32, 474-480.

Huxlin, K. R., Martin, T., Kelly, K., Riley, M., Friedman, D. I., Burgin, W. S., \& Hayhoe, M. (2009). Perceptual relearning of complex visual motion after V1 damage in humans. J Neurosci, 29, 3981-3991.

Jeter, P. E., Dosher, B. A., Petrov, A., \& Lu, Z. L. (2009). Task precision at transfer determines specificity of perceptual learning. J Vis, 9, 1 1-13. 
Karni, A., \& Sagi, D. (1991). Where practice makes perfect in texture discrimination: evidence for primary visual cortex plasticity. Proc Natl Acad Sci U S A, 88, 49664970.

Kasten, E., Wust, S., Behrens-Baumann, W., \& Sabel, B. A. (1998). Computer-based training for the treatment of partial blindness. Nat Med, 4, 1083-1087.

Kraft, A., Roehmel, J., Olma, M. C., Schmidt, S., Irlbacher, K., Brandt, S. A. (2010). transcranial direct current stimulation affects visual perception measured by threshold perimetry. Exp Brain Res, 207, 283-290.

Kuai, S. G., Zhang, J. Y., Klein, S. A., Levi, D. M., \& Yu, C. (2005). The essential role of stimulus temporal patterning in enabling perceptual learning. Nat Neurosci, 8, 1497-1499.

Kumano, H., \& Uka, T. (2013). Neuronal mechanisms of visual perceptual learning. Behav Brain Res, 249, 75-80.

Levi, D. M., \& Li, R. W. (2009). Perceptual learning as a potential treatment for amblyopia: a mini-review. Vision Res, 49, 2535-2549.

Levitt, H. (1971). Transformed up-down methods in psychoacoustics. J Acoust Soc Am, 49, Suppl 2:467+.

Li, R. W., Young, K. G., Hoenig, P., \& Levi, D. M. (2005). Perceptual learning improves visual performance in juvenile amblyopia. Invest Ophthalmol Vis Sci, 46, 31613168.

Liu, Z., \& Weinshall, D. (2000). Mechanisms of generalization in perceptual learning. Vision Res, 40, 97-109.

Looi, C. Y., Duta, M., Brem, A. K., Huber, S., Nuerk, H. C., \& Cohen Kadosh, R. (2016). Combining brain stimulation and video game to promote long-term transfer of learning and cognitive enhancement. Sci Rep, 6, 22003.

Lu, Z. L., Liu, J., \& Dosher, B. A. (2010). Modeling mechanisms of perceptual learning with augmented Hebbian re-weighting. Vision Res, 50, 375-390.

Mancuso, L. E., Ilieva, I. P., Hamilton, R. H., \& Farah, M. J. (2016). Does Transcranial Direct Current Stimulation Improve Healthy Working Memory?: A Meta-analytic Review. J Cogn Neurosci, 7, 1-27.

Maniglia, M., Pavan, A., Cuturi, L. F., Campana, G., Sato, G., \& Casco, C. (2011). Reducing crowding by weakening inhibitory lateral interactions in the periphery with perceptual learning. PLoS One, 6, e25568.

Marshall, L., Molle, M., Hallschmid, M., \& Born, J. (2004). Transcranial direct current stimulation during sleep improves declarative memory. J Neurosci, 24(44), 99859992.

McGovern, D. P., Webb, B. S., \& Peirce, J. W. (2012). Transfer of perceptual learning between different visual tasks. J Vis, 12, 4.

Miniussi, C., Harris, J.A., \& Ruzzoli, M. (2013). Modelling non-invasive brain stimulation in cognitive neuroscience. Neurosci Biobehav Rev, 37(8), 1702-1712.

Monti, A., Cogiamanian, F., Marceglia, S., Ferrucci, R., Mrakic-Sposta, S., Vergari, M., Zago, S., \& Priori, A. (2008). Improved naming after transcranial direct current stimulation in aphasia. J Neurol Neurosurg Psychiatry, 79, 451-453.

Moss, F., Ward, L. M., \& Sannita, W. G. (2004). Stochastic resonance and sensory information processing: a tutorial and review of application. Clin Neurophysiol, 115, 267-281.

Nitsche, M. A., Kuo, M. F., Karrasch, R., Wachter, B., Liebetanz, D., \& Paulus, W. (2009). Serotonin affects transcranial direct current-induced neuroplasticity in humans. Biol Psychiatry, 66, 503-508. 
Nitsche, M. A., \& Paulus, W. (2000). Excitability changes induced in the human motor cortex by weak transcranial direct current stimulation. J Physiol, 527(3), 633639.

Pelli, D. G. (1997). The VideoToolbox software for visual psychophysics: transforming numbers into movies. Spat Vis, 10, 437-442.

Petrov, A. A., Dosher, B. A., \& Lu, Z. L. (2005). The dynamics of perceptual learning: an incremental reweighting model. Psychol Rev, 112, 715-743.

Pirulli, C., Fertonani, A., \& Miniussi, C. (2013). The role of timing in the induction of neuromodulation in perceptual learning by transcranial electric stimulation. Brain Stimul, 6, 683-689.

Pisoni, A., Papagno, C., \& Cattaneo, Z. (2012). Neural correlates of the semantic interference effect: new evidence from tDCS. Neuroscience, 223C, 56-67.

Plank, T., Rosengarth, K., Schmalhofer, C., Goldhacker, M., Brandl-Ruhle, S., \& Greenlee, M. W. (2014). Perceptual learning in patients with macular degeneration. Front Psychol, 5, 1189.

Polat, U. (2009). Making perceptual learning practical to improve visual functions. Vision Res, 49, 2566-2573.

Polat, U., Ma-Naim, T., Belkin, M., \& Sagi, D. (2004). Improving vision in adult amblyopia by perceptual learning. Proc Natl Acad Sci U S A, 101, 6692-6697.

Polat, U., Schor, C., Tong, J. L., Zomet, A., Lev, M., Yehezkel, O., Sterkin, A., \& Levi, D. M. (2012). Training the brain to overcome the effect of aging on the human eye. Sci Rep, 2, 278.

Ponomarenko, A. A., Li, J. S., Korotkova, T. M., Huston, J. P., \& Haas, H. L. (2008). Frequency of network synchronization in the hippocampus marks learning. Eur J Neurosci, 27, 3035-3042.

Poreisz, C., Boros, K., Antal, A., \& Paulus, W. (2007). Safety aspects of transcranial direct current stimulation concerning healthy subjects and patients. Brain Res Bull, 72, 208-214.

Rosengarth, K., Keck, I., Brandl-Ruhle, S., Frolo, J., Hufendiek, K., Greenlee, M. W., \& Plank, T. (2013). Functional and structural brain modifications induced by oculomotor training in patients with age-related macular degeneration. Front Psychol, 4, 428.

Ross, L., McCoy, D., Wolk, D. A., Coslett, B., \& Olson, I. R. (2010). Improved proper name recall by electrical stimulation of the anterior temporal lobes. Neuropsychologia 48(12), 3671-3674.

Sabel, B. A., Kenkel, S., \& Kasten, E. (2005). Vision restoration therapy. Br J Ophthalmol, $89,522-524$.

Sagi, D. (2011). Perceptual learning in Vision Research. Vision Res, 51, 1552-1566.

Sale, A., De Pasquale, R., Bonaccorsi, J., Pietra, G., Olivieri, D., Berardi, N., \& Maffei, L. (2011). Visual perceptual learning induces long-term potentiation in the visual cortex. Neuroscience, 172, 219-225.

Sandrini, M., Brambilla, M., Manenti, R., Rosini, S., Cohen, L. G., \& Cotelli. M. (2014). Noninvasive stimulation of prefrontal cortex strengthens existing episodic memories and reduces forgetting in the elderly. Front Aging Neurosci, 6:289.

Silvanto, J., Muggleton, N., \& Walsh, V. (2008). State-dependency in brain stimulation studies of perception and cognition. Trends Cogn Sci, 12, 447-454.

Sparing, R., Dafotakis, M., Meister, I. G., Thirugnanasambandam, N., \& Fink, G. R. (2008). Enhancing language performance with noninvasive brain stimulation--a transcranial direct current stimulation study in healthy humans. Neuropsychologia, 46, 261-268. 
Spiegel, D. P., Byblow, W. D., Hess, R. F., \& Thompson, B. (2013). Anodal transcranial direct current stimulation transiently improves contrast sensitivity and normalizes visual cortex activation in individuals with amblyopia. Neurorehabil Neural Repair, 27, 760-769.

Stagg, C. J., Best, J. G., Stephenson, M. C., O'Shea, J., Wylezinska, M., Kincses, Z. T., Morris, P. G., Matthews, P. M., \& Johansen-Berg, H. (2009). Polarity-sensitive modulation of cortical neurotransmitters by transcranial stimulation. J Neurosci, 29, 52025206.

Schwarzkopf, D. S, Silvanto, J., \& Rees, G. (2011). Stochastic resonance effects reveal the neural mechanisms of transcranial magnetic stimulation. J Neurosci, 31(9), 31433147.

Tan, D. T., \& Fong, A. (2008). Efficacy of neural vision therapy to enhance contrast sensitivity function and visual acuity in low myopia. J Cataract Refract Surg, 34, 570-577.

Terney, D., Chaieb, L., Moliadze, V., Antal, A., \& Paulus, W. (2008). Increasing human brain excitability by transcranial high-frequency random noise stimulation. $J$ Neurosci, 28, 14147-14155.

Thompson, B., Mansouri, B., Koski, L., \& Hess, R. F. (2008). Brain plasticity in the adult: modulation of function in amblyopia with rTMS. Curr Biol, 18, 1067-1071.

Tyler, C. W. (1997). Colour bit-stealing to enhance the luminance resolution of digital displays on a single pixel basis. Spat Vis, 10, 369-377.

Vannorsdall, T. D., van Steenburgh, J. J., Schretlen, D. J., Jayatillake, R., Skolasky, R. L., \& Gordon, B. (2016). Reproducibility of tDCS Results in a Randomized Trial: Failure to Replicate Findings of tDCS-Induced Enhancement of Verbal Fluency. Cogn Behav Neurol, 29(1),11-7. Webb, B. S., Roach, N. W., \& McGraw, P. V. (2007). Perceptual learning in the absence of task or stimulus specificity. PLoS One, 2, e1323.

Xiao, L. Q., Zhang, J. Y., Wang, R., Klein, S. A., Levi, D. M., \& Yu, C. (2008). Complete transfer of perceptual learning across retinal locations enabled by double training. Curr Biol, 18, 1922-1926.

Yun, G. J., Chun, M. H., \& Kim, B. R. (2015). The Effects of Transcranial Direct-Current Stimulation on Cognition in Stroke Patients. J Stroke, 17, 354-358.

Zhou, Y., Huang, C., Xu, P., Tao, L., Qiu, Z., Li, X., \& Lu, Z. L. (2006). Perceptual learning improves contrast sensitivity and visual acuity in adults with anisometropic amblyopia. Vision Res, 46, 739-750. 